\title{
Aspectos radiológicos da tuberculose primária da mama: relato de caso e revisão de literatura
}

\author{
Radiological aspects of primary tuberculosis in the breast: \\ a case report and review of the literature
}

\author{
Sabrina Ramos Bianco ${ }^{1}$, Rafael Lopes Gurgel ${ }^{2}$ \\ e Michel de Araújo Tavares ${ }^{3}$
}

\section{RESUMO}

A tuberculose mamária é uma enfermidade equivalente a menos que 0,1\% das lesões deste órgão. Apresentamos a seguir um caso de nosso serviço discutindo os achados radiológicos da mamografia, ultrassonografia e ressonância magnética, incluindo curvas cinéticas. A tuberculose mamária é uma condição ímpar, sendo importante o relato pelos diagnósticos diferenciais com carcinoma mamário e o abscesso piogênico.

Palavras-chaves: Tuberculose. Mama. Infecção. Radiologia.

\section{ABSTRACT}

Mammary tuberculosis is an illness accounting for less than $0.1 \%$ of breast lesions. We present a case from our service and discuss the radiological findings from mammography, ultrasound scan and magnetic resonance, including kinetic curves. Mammary tuberculosis is an unusual condition and such reports are important in relation to the differential diagnoses versus breast cancer and pyogenic abscess.

Key-words: Tuberculosis. Breast. Infection. Radiology.

A infecção pelo Mycobacterium tuberculosis é uma enfermidade clínica em países em desenvolvimento, estando em declínio em nações desenvolvidas. Contudo, nas décadas de 80 e 90, tivemos um retorno da prevalência da doença pela epidemia de HIV/AIDS associado a sua multirresistência. As manifestações extrapulmonares acontecem em poucos casos. A tuberculose mamária é uma condição rara tendo importância pelo diagnóstico diferencial com outras patologias deste orgão ${ }^{1}$.

Apresenta uma incidência menor que $0,1 \%$ das lesões mamárias analisadas histologicamente ${ }^{24}$. A forma nodular pode simular um carcinoma ou abscesso piogênico ${ }^{3}$. A presença de reação granulomatosa pode ter várias hipóteses histológicas, tais como: aspergilose e esquistossomose ${ }^{3}$.

0 diagnóstico de tuberculose extrapulmonar é feito pelas evidências clínicas, achados laboratoriais sugestivos das formas ativas em pacientes com pelo menos uma cultura positiva para Mycobacterium tuberculosis de material extrapulmonar ${ }^{4}$.

De acordo com a literatura, os achados de tuberculose mamária são raros e os relatos na área radiológica não são

1. Setor de Ultrasonografia e Mamografia, Clínica Sensumed, Manaus, AM, Brasil. 2. Escola Superior de Ciências da Saúde, Universidade do Estado do Amazonas, Manaus, AM, Brasil. 3. Setor de Tomografia Computadorizada e Ressonância Magnética, Clínica Sensumed, Manaus, AM, Brasil.

Endereço para correspondência: Dra Sabrina Bianco. Clínica Sensumed. Rua São Luis 510, Bairro Adrianópolis, 69057-259 Manaus, AM.

Tel: 5592 2129-5600; Fax: 5592 2129-5610

e-mail: sabrinabianco@bol.com.br

Recebido para publicação em 22/07/2008

Aceito em 20/03/2009 muito extensos. Procuramos neste relato analisar e descrever os achados de imagem na mamografia (MMG), ultrassonografia (US) e ressonância magnética (RM) em paciente acometida de tuberculose primária da mama.

A paciente foi submetida a exame clínico, teste do derivado purificado da proteína (PPD), biópsia das lesões com exame histopatológico e exames de imagem incluindo radiografia de tórax, MMG, US e RM, neste último incluindo seqüências ponderadas em T1 e T2 com e sem supressão de gordura no plano sagital e por fim uma sequiência dinâmica pós-contraste de 1 a 5 minutos. Esta enfermidade foi suspeitada pelo quadro clínico insidioso, não tendo sido encontrados outros focos de infecção de tuberculose. A paciente recebeu esquema tríplice tuberculostático com a terapêutica sendo iniciada empiricamente. 0 seguimento foi feito durante 6 meses por meio de consulta ambulatorial mensal na Fundação de Medicina Tropical do Amazonas.

\section{RELATO DO CASO}

A paciente do sexo feminino, 32 anos, casada, parda, três filhos, em uso de anticoncepcionais orais por 10 anos, relatou tratamento prévio para papilomavírus humano (HPV), tendo realizado sorologia para HIV e sífilis, sendo não reagentes.

A paciente encontrava-se em astenia geral e anorexia, apresentando perda de $5 \mathrm{~kg}$ em três meses. Negou casos de tuberculose na família e contato prévio. A paciente possuía sintomas de dor e endurecimento na mama esquerda que não 
melhorava com o uso de antiinflamatório oral, sendo então solicitados exames radiológicos das mamas.

A radiografia de tórax não demonstrou sinais sugestivos de processo específico. A mamografia evidenciou a presença de focos de tecido fibroglandular com aumento da densidade no quadrante súpero-lateral e retroareolar da mama esquerda (Figura 1).

Na ultrassonografia, observou-se presença de nódulo sólido, hipoecóico, heterogêneo, de contornos macrolobulados com vascularização de permeio (Figura 2), sendo então realizado biópsia. 0 exame histopatológico indicou processo inflamatório, de distribuição perilobular com linfócitos e histiócitos epitelióides com formação de granulomas, mostrando-se mastite lobular granulomatosa.
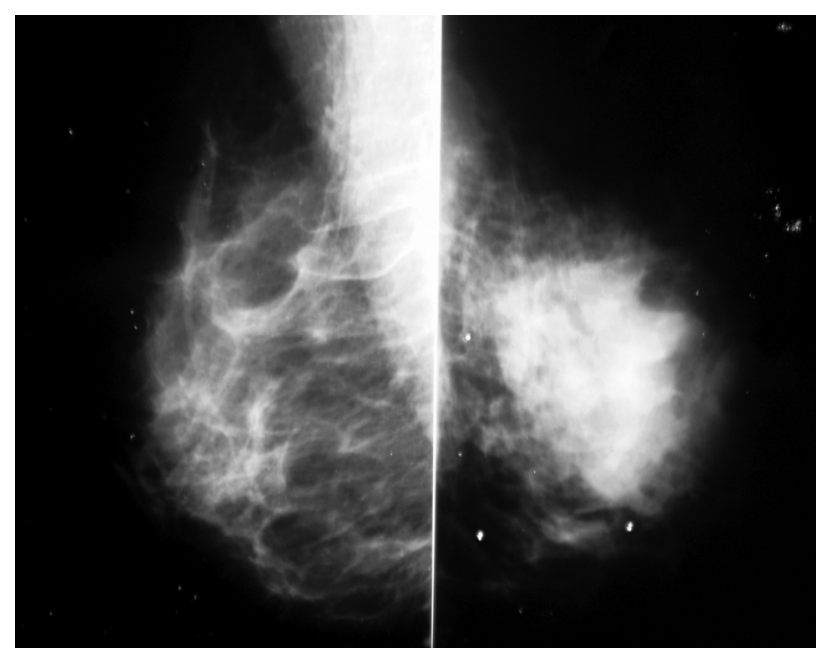

FIGURA 1

Mamografia na incidência médio lateral oblíqua demonstrando aumento da densidade do parênquima mamário no quadrante súpero-lateral e retroareolar da mama esquerda.

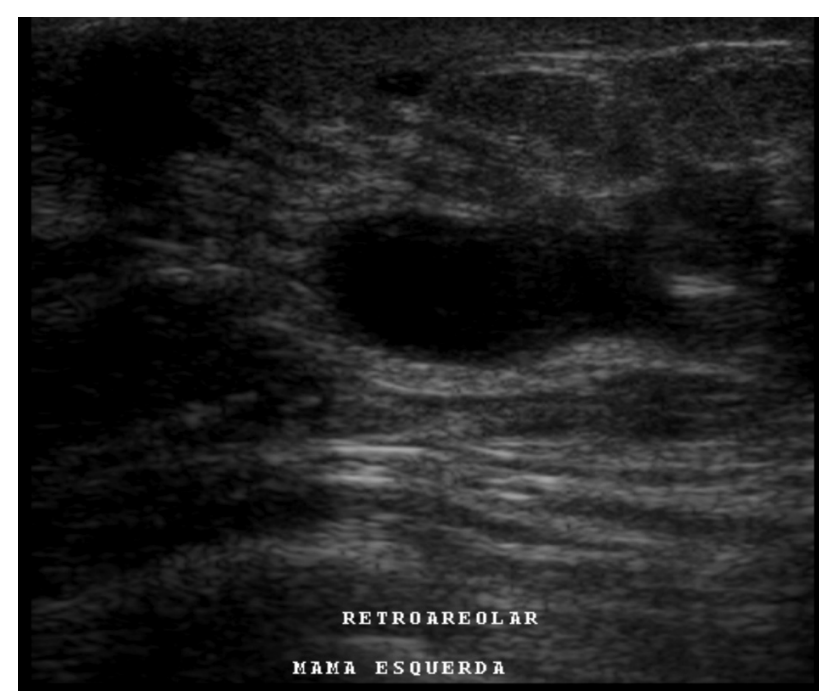

FIGURA 2

Ultrassonografia mamária demonstrando lesão nodular de aspecto heterogêneo na região retroareolar da mama esquerda.
Foi realizada RM para investigar o tamanho da massa e procurar lesões adicionais na mama afetada. As diversas sequiências e imagens obtidas nos planos axiais e sagitais demonstraram lesão nodular sólida e contornos levemente lobulares, definidos medindo cerca de $1,2 \mathrm{~cm}$ que sofrem impregnação anômala pelo meio de contraste paramagnético associado a discreta distorção da arquitetura do parênquima mamário adjacente (Figura 3). Foi realizado estudo complementar com curva de análise quantitativa e qualitativa de impregnação de contraste.

Pelos aspectos clínicos, radiográficos e histopatológicos foi iniciado o esquema tríplice tuberculostático empiricamente, evoluindo com a regressão do nódulo e melhora do estado geral (Figura 4).

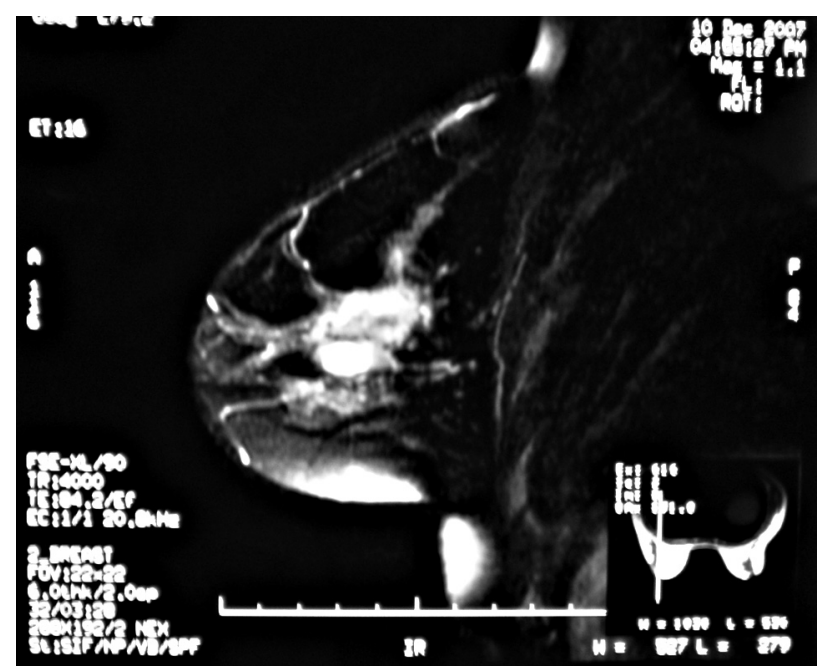

FIGURA 3

Ressonância magnética mamária na sequiência ponderada em T2 com supressão de gordura no plano sagital demonstrando lesão nodular sólida de contornos lobulados caracterizada por hipersinal da região retroareolar na mama esquerda.

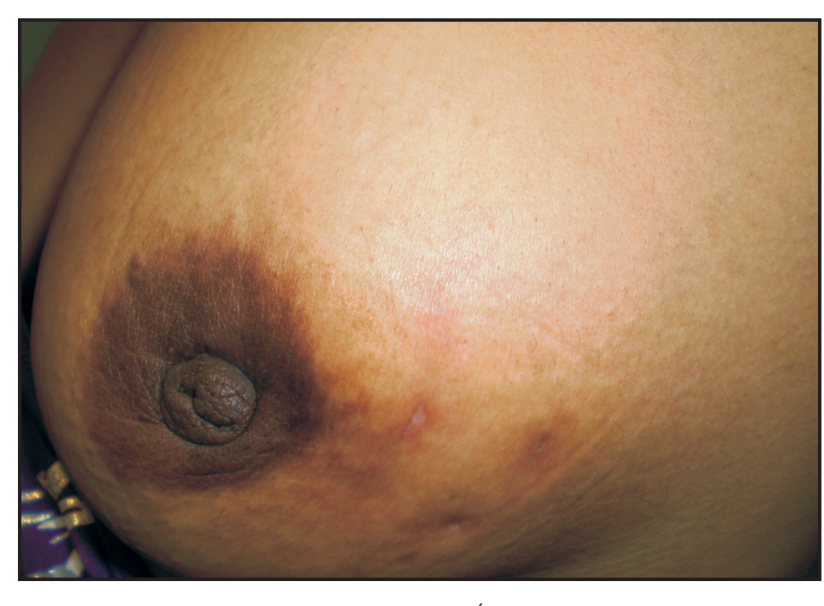

FIGURA 4

Paciente após início do tratamento demonstrando área de retração cutânea de fístulas cicatrizadas em quadrante ínfero-lateral.

\section{DISCUSSÃO}

A tuberculose é uma infecção comum, contudo o envolvimento das mamas é uma condição rara e descrita desde o século XIX 5 . Tal enfermidade perfaz 0,1\% das patologias mamárias nos países 
desenvolvidos e 3 a 4,5\% em países em desenvolvimento ${ }^{26}$. Com o surto do HIV/AIDS no mundo, houve um aumento dos casos de tuberculose extrapulmonar em imunodeprimidos em até $60 \%{ }^{7}$. Há poucos relatos na literatura sobre a aparência mamográfica, ultrassonográfica da mastite granulomatosa idiopática (MGI).

Os sintomas e sinais concordam com a literatura, mostrando dor mamária, presença de nódulo e fístulas com drenagem cutânea ${ }^{257}$. Segundo Tewari, a MMG evidência como característica mais comum aspecto nodular de contornos indefinidos que pode ser confundido com carcinoma mamário ${ }^{4}$. A ultrassonografia demonstra a lesão nodular, heterogênea e hipoecóica que vai ao encontro da literatura ${ }^{35}$.

Em nosso trabalho, não foi visualizado o Mycobacterium tuberculosis no histopatológico, mas relato de uma série de 14 casos de tuberculose mamária em que o bacilo de Koch foi isolado também em apenas um caso ${ }^{10}$.

0 diagnóstico diferencial com mastite granulomatosa idiopática acomete mulheres jovens, com idade variando entre 17 e 42 anos (média de 33 anos), e relação com gravidez e lactação, possuindo os achados mamográficos com pequenos nódulos mal definidos, densidades assimétricas extensas e distorção do tecido mamário sem microcalcificações, linfonodos axilares aumentados. 0 aspecto ultrassonográfico de múltiplas imagens tubulares hipoecóicas e heterogêneas, freqüentemente contíguas e confluentes como fortemente sugestivas de MGI ${ }^{11}$. 0 carcinoma de mama é o principal diagnóstico diferencial nas suas formas nodular e inflamatória, podendo entrar no diagnóstico também: abscesso mamário, necrose gordurosa, actinomicose e infecções fúngicas, mas a presença de uma clínica sugestiva e um histopatológico são importantes para guiar na terapêutica ${ }^{10}$.

A ressonância magnética foi executada para avaliar o tamanho da massa e procurar lesões adicionais. Foi realizada a sequiência T1 com supressão de gordura no plano axial em fase dinâmica de 1 a 5 minutos, onde se observou área de impregnação nodular numa fase precoce e notadamente tardia com curva do tipo II (em platô). No entanto, demonstrando dificuldade de diferenciação entre carcinoma mamário e abscesso piogênico ${ }^{1012}$.

0 estudo de análise da curva é realizado após administração de meio de contraste paramagnético e pode ser classificada: Tipo I (ascendente), tipo II (platô) e tipo III wash-out ${ }^{7}$.
Enquanto a RM parece ter alta sensibilidade para a detecção de câncer de mama, nem todas as lesões captantes de contraste são malignos ${ }^{10}$. No caso relatado, houve impregnação ao meio de contraste, no entanto provou-se ser doença granulomatosa.

Concluindo, o quadro clínico associado a análise radiológica deste relato demonstrou que os diversos métodos de imagem podem ser uma ferramenta valiosa para o diagnóstico da tuberculose de mama. As características radiológicas não são específicas para este processo infeccioso. No entanto, a RM pode ser uma opção para complementação do diagnóstico diferencial do carcinoma e do abscesso mamário, entrando neste estudo a análise de curvas de impregnação de contraste que podem colaborar com o diagnóstico diferencial e para analisar a extensão da lesão extramamária, devendo-se ter maiores estudos neste tema.

\section{REFERÊNCIAS}

1. Fanlo P, Tiberio G. Extrapulmonary tuberculosis. Anales del Sistema Sanitario de Navarra 2: 143-162, 2007.

2. Fellah L, Leconte I, Weynand B, Donnez J, Berlière M. Breast tuberculosis imaging. Fertility and Sterility 2: 460-461, 2006.

3. Jah A, Mulla R, Lawrence FD, Pittam M, Ravichandran D. Tuberculosis of the breast: experience of a UK breast clinic serving an ethnically diverse population. Annals of The Royal College of Surgeons of England, p. 416-419, 2004.

4. Kervancioglu S. Primary tuberculosis of the breast: Diagnostic and Interventional Radiology 4: 210-212, 2005.

5. Khanna R, Prasanna GV, Gupta P, Kumar M, Khanna S, Khanna AK. Mammary tuberculosis: report on 52 cases. Postgraduate Medical Journal 921: 422-424, 2002.

6. Mirsaeidi SM, Masjedi MR, Mansouri SD, Velayati AA. Tuberculosis of the breast: report of 4 clinical cases and literature review. Eastern Mediterranean Health Journal 3: 670-676, 2007.

7. Morris EA, Liberman L. Breast MRI. Diagnosis and intervention. Springer, Estados Unidos, 1996.

8. Orel SG, Hochman MG, Schwall MD, Reynolds C, Sullivan DC. High-resolution MR Imaging of the breast: clinical context. Radiographics 16: 1385-1401, 1996.

9. Sabate JM. Radiologic evaluation of uncommon inflammatory and reactive breast disorders. Radiographics 2: 411-424, 2005.

10. Silva ERD, Cavalcante EF, Gomes EF, Ferreira FVA, Soares LRC, Furtado GHC, Juaçaba SF. Tuberculose Primária da Mama. Revista Brasileira de Ginecologia e Obstetrícia 24: 241-246, 2002.

11. Stefanon CC, Gonçalves AF, Lima R, Rossi K. mastite granulomatosa idiopática: aspectos clínicos, radiológicos e ultra-sonográficos 3: 225-230, 2005.

12. Tewari M, Shukla HS, Breast tuberculosis: diagnosis, clinical features \& management. Indian Journal of Medical Research 2: 103-110, 2005. 